\title{
On the wave-particle interaction and removal of energetic particles
}

\author{
Umesh P. Singh \\ Dept. of Physics, Seth Jai Parkash Mukand Lal Institute of Engineering and Technology, \\ Radaur 135133, Yamunanagar, Haryana, India. \\ Corresponding author:dr.umeshsingh@gmail.com
}

\begin{abstract}
There is always a risk of destruction of man-made satellites by the energetic electrons trapped in Van Allen radiation belts in space. These energetic electrons also pose a biological danger to astronauts. The cyclotron resonance interaction is studied between the whistler-mode waves in the frequency range of ELF (Extremely Low Frequency $300-3000 \mathrm{HZ}$ ) and VLF (Very Low Frequency $3-30 \mathrm{kHz}$ ) propagating along geomagnetic field line and counter streaming energetic electron. During this process the pitch angle of energetic electrons reduces. This results in the dumping of these electrons into the lower ionosphere. This makes electrons unable to strike the satellites orbiting in low Earth orbit, Geosynchronous, Sun-synchronous or polar orbit. It is shown that the lifetime values of energetic electrons vary from 2.03 to 227.68 hours at low latitudes. It is shown that these waves can remove these energetic electrons from their path and ensure the safety of satellites.
\end{abstract}

Keywords: Energetic electrons, particles precipitation, trapped particles, wave-particle interaction, whistler-mode.

\section{Introduction}

Since the launch of the artificial satellite "Sputnik" in 1957 by the USSR, the unique space probing technique has been effectively used for ionospheric and upper atmospheric studies, employing a wide range of electromagnetic spectrums. Later on, the satellites were used to probe the upper ionosphere and inner plasmasphere/ magnetosphere and these satellites helped to collect information about a large number of parameters such as electron density, ion composition, electric field, etc (Singh, 1993). These satellites are used for various purposes like remote sensing, point-to-point communication on earth, space, planes, and vehicles, and television and radio broadcasting.

The Low Earth Orbit (LEO) is a circular orbit that is about $400 \mathrm{~km}$ above the earth's surface and, correspondingly, a period of about 90 minutes. Being at a low altitude, these satellites are visible from within a radius of approximately $1000 \mathrm{~km}$ from the sub-satellite point. CubeSats are miniature satellites that are commonly used in low Earth orbit for applications such as remote sensing or communications

The satellite in an elliptical orbit is highly inclined, ensuring good elevation over selected positions during the northern portion of the orbit. The Molniya satellites which are present in highly inclined orbits are used for telephony and TV services over Russia.

The geostationary satellite appears to be in a fixed position to an observer on the earth. The time taken in one revolution by such a satellite is equivalent to the time taken by the earth in one revolution. The geostationary orbit is most useful for communication applications for groundbased antennas, which are directed towards the satellite. It can operate effectively without any installation of expensive equipment to track the satellite's motion. The first truly geostationary satellite launched in orbit was 
the Syncom 3, launched on August 19, 1964, at $180^{\circ}$ East longitude.

A possible source of risk to these satellites is the presence of highly energetic particles like electrons which may lead satellites to malfunction. These very high energy particles are termed as 'killer electrons' in the outer atmosphere of the earth (Chen et al., 2007). These are strong enough to degrade the performance of electronic devices in the spacecraft and could damage human tissues and cause biological hazardous to the person onboard the spacecraft or satellite. For many years, the mechanism by which they are produced has remained poorly understood, despite physicists' attempts at solving this puzzle. It would be a big step towards understanding the physics of the Van Allen radiation belts and could pave the way to ensure the safety of satellites in space. Thus, such reports would be valuable for satellites and telecommunication systems, because storms in the radiation belts disrupt communications and may damage satellites. The energetic electrons in the radiation belts are very dangerous to satellites. These are connected to the increased intensity of solar winds, called geomagnetic storms, and are associated with magnificent auroral displays over the poles. But until now there was no definitive understanding of how relatively harmless electrons get accelerated to become dangerous electrons.

If an electron in the radiation belt happens to be wobbling at the same rate, it will gain energy and accelerate across the magnetic field. The electromagnetic waves may be the main cause of killer electrons. The low-frequency waves injected into the lower radiation belt could cause the very high-energy electrons to rain out into the atmosphere, ending a month-long geomagnetic storm effectively in a matter of days. This can be done by the wave-particle interaction between ELF/VLF waves and counter-streaming very energetic electrons (Inan, 1977).

The wave-particle interaction between highly energetic electrons and whistler-mode
ELF/VLF waves which normally propagate parallel to the geomagnetic field lines plays a very important role in the pitch angle scattering of these energetic electrons into the lower ionosphere. The processes may be cyclotron resonance (pitch angle of the electron is 900), Cherenkov resonance (pitch angle of the electron is 00), Coulomb scattering (describes the interaction between two charged particles), and many other processes.

Herein, we will consider the cyclotron resonance because the ELF / VLF waves while propagating along geomagnetic field lines interact with counter-streaming energetic electrons having perpendicular velocity components. During waveparticle, interaction electrons experience an approximately stationary wave field for an extended period resulting in energy exchange with wave field thereby causing energy and pitch angle diffusion of electrons. Various phenomena like aurora, X-rays, Bremsstrahlung and perturbation in D and $\mathrm{E}$ region may take place (Chang \& Inan, 1983; Helliwell, 1965; Inan, 1977; Singh, 1991; Singh \& Singh, 2006).

The radiation belt surrounding the earth has trapped energetic electrons which enter the belt after emerging from the Sun, and are known as the solar particles. Because these particles gyrate around field lines coming from north to south, and south to north, traversing both times the equatorial region, they remain trapped in the radiation belt for a long time till they are de-energized after many rotations from one hemisphere to the opposite one (Potapov et al., 2016; Daglis et al., 2019). Another method may be wave-particle interaction to de-energize such particles. In this case, the energy of the energetic particles is reduced and their pitch angle decreases.

The radiation belts are of two types. First one is called the inner radiation belt $(\mathrm{L}=1.1$ or 1.2 to $L=1.7$ or 1.8 ) and the second one is called outer radiation belt $(\mathrm{L}=2.2$ or 2.4 to 7 or 8$)$. Innerbelt covers the low latitude region of $\lambda=17.550$ to $\lambda=41.80$. But generally, the 
low latitude region is considered up to 350 - 380 only and the middle latitude region is considered from 400 and above. The position of the inner/outer boundaries of the radiation belts depends upon the $\mathrm{Kp}$ index, relating to the disturbance of the Earth's magnetic field caused by the solar wind. The faster the solar wind blows, the greater is the turbulence. The index ranges from 0 , for low activity, to 9, which means that an intense geomagnetic storm is underway. The inner radiation belt has much greater importance as the communication satellites rotate in this region $(250-750 \mathrm{~km})$. Considering that a communication satellite is a costly and very important instrument for smooth and continuous signal transmission, its safety must be our utmost priority. This paper has studies the wave-particle interaction between ELF/VLF waves which are propagating parallel to the geomagnetic field and highly energetic particles trapped in the inner radiation belt.

\section{Electron density and method of calculation}

The electron densities which are employed here are taken from the Diffusive Equilibrium (DE) model of Angerami and Thomas (1963), shown in Table 1.

Table 1. Variation of electron density with

\begin{tabular}{|cccccccc|}
\multicolumn{1}{c|}{ L value } \\
\hline $\mathrm{L}$ & 1.1 & 1.2 & 1.3 & 1.4 & 1.5 & 1.6 & 1.7 \\
\hline $\begin{array}{l}\mathrm{N}(\mathrm{el} / \\
\left.\mathrm{cm}^{3}\right)\end{array}$ & 20,000 & 8130 & 4610 & 3390 & 2710 & 2290 & 1980 \\
\hline
\end{tabular}

This model is represented at a reference level of $400 \mathrm{~km}$ by an electron density of $15 \times 104 \mathrm{el} / \mathrm{cm}^{3}$,

$\mathrm{O}+$ of $95 \%$,

$\mathrm{H}+$ of $0.25 \%$,

$\mathrm{He}+$ of $4.75 \%$ with temperature of 1000

The density in this DE Model is taken from Alouette-1 observations corresponding to nighttime, the oxygen and helium densities as well as temperature are taken from the incoherent backscattered spectra gathered nighttime hydrogen ions concentration is taken from Injun-3 proton whistler analysis (Prasad, 1968). This model has been used earlier by (Singh \& Singh, 1998; Vranjes \& Tanaka, 2005; Bortnik et. al., 2011).

We know that plasmapause is the location in the magnetosphere where electron density suddenly falls to $1 / 10$ or $1 / 20$ of the general value. The plasmapause location depends upon the $\mathrm{K}_{\mathrm{p}}$ index which depends upon 24 hours average value of the Geomagnetic Field. $\mathrm{K}_{\mathrm{p}}$ is an excellent indicator of disturbances in the Earth's magnetic field and is used by Space Weather Prediction Center, USA to decide whether geomagnetic alerts and warnings need to be issued for users who are affected by these disturbances. The principal users affected by geomagnetic storms are the electrical power grid, spacecraft operations, users of radio signals that reflect off of or pass through the ionosphere, and observers of the aurora. The plasmapause locations $\left(\mathrm{L}_{\mathrm{PT}}\right)$ are related with $\mathrm{K}_{\mathrm{p}}$ as $-\mathrm{L}_{\mathrm{PT}}=5.6-0.46 \mathrm{xK}_{\mathrm{p}}$.

To compute the pitch-angle diffusion coefficient, $\mathrm{D}(\alpha)$, we use the following expression (Sentman\& Goertz, 1978)

$$
D(\alpha)=2 \omega_{H e}^{2} \frac{B_{w}^{2}}{B_{0}^{2}} \frac{1}{E_{n}\left(E_{n}^{2}-1\right)^{1 / 2} \mu \Delta \omega}
$$

Or,

$$
D(\alpha)=2\left(2 \pi f_{H e}\right)^{2} \frac{B_{w}^{2}}{B_{0}^{2}} \frac{1}{E_{n}\left(E_{n}^{2}-1\right)^{1 / 2} Q}
$$

Where $Q=\mu \cdot 2 \pi \Delta f . B_{w}$ is the magnetic amplitude of the whistler-mode wave. Here

$\omega_{H e}$ is electrons gyro frequency which can be written as

$$
\omega_{H e}=2 \pi f_{H e}=\frac{e B}{m_{0} c}
$$

In the equation above, $\mathrm{e}$ is the electronic charge, $\mathrm{B}$ is the geomagnetic field at a given position in space/surface on the earth's surface and $m_{o}$ and $c$ are the rest mass of electron and velocity of light in vacuum. En is normalized resonant energy of the electron, i.e. resonant energy $E_{r}$ is 
divided by $511 \mathrm{keV}$. Equation 3, in brief, can be written as (Singh, 1992)

$$
f_{H e}(k H z)=\frac{873.6}{L^{3}}
$$

In Equation 1, refractive index $\mu$ is measured using the following expression. (Inan, 1977; Inan et al., 1978)

$$
\mu^{2}=1+\left[\frac{\omega_{P_{e}}^{2}}{\omega\left(\omega_{H e}-\omega\right)}\right]
$$

Where $\omega_{P e}$ is electron's plasma frequency expressed as

$$
\omega_{p_{e}}=2 \pi f_{P_{c}}
$$

Here $f_{P_{e}}^{2}=80.63 x N$ (Singh \& Singh, 2004), $\mathrm{N}$ is electron's density in electrons per $\mathrm{cm}^{3}$ and $f_{P e}$ is in $\mathrm{kHz} . \Delta \omega$ is nothing but wave bandwidth i.e. $\Delta \omega=2 \pi \Delta \mathrm{f}$, where $\mathrm{f}$ is in $\mathrm{Hz}$. In this case, we consider frequencies of 1,2 , $3,4,5 \mathrm{kHz}$ at $\mathrm{L}$ values of low, mid, and high L shells, in which $1-3 \mathrm{kHz}$ represent ELF waves and $3-5 \mathrm{kHz}$ are representative of VLF waves.

As far as geomagnetic field $\mathrm{B}$ is concerned we can use the following formula for it (Inan, 1977)

$$
B=\frac{B_{0}}{L^{3} \cdot\left(1+3 \sin ^{2} \lambda\right)^{1 / 2}}
$$

Where B0 is the magnetic field at earth surface written as

$$
\mathrm{B}_{0}=0.314 \times 10^{-4} \text { (in Tesla) }
$$

At the equator, $\lambda$ is zero. So the expression for the $\mathrm{B}$ at the geomagnetic equator for different locations of a given geomagnetic field line where wave-particle interaction generally occurs can be written as

$$
B=\frac{0.314 \times 10^{-4}}{L^{3}}(\text { inTesla })
$$

$\mathrm{L}$, the McIlwain parameter is expressed as (Inan, 1977; Singh, 1991)

$$
L=\frac{1}{\cos ^{2} \lambda}=\frac{R}{R_{0}}
$$

$\mathrm{R}$ is the geocentric distance of any region in space and R0 is Earth radius (6372 km). It is well known that $B_{f}^{2}=\frac{B_{w}^{2}}{\Delta f}$ Equation 1 given for diffusion coefficient $\mathrm{D}(\alpha)$ can be written now as

$$
D(\alpha)=\frac{9.734 \times 10^{-3}}{E_{n}\left(E_{n}^{2}-1\right)^{1 / 2} \mu}
$$

The inverse of diffusion coefficient $D(\alpha)$ is called the lifetime of the energetic resonant/ killer electron.

$$
\text { Life Time }=\frac{1}{D(\alpha)}
$$

\section{Results and discussion}

\subsection{Loss cone pitch angle}

It is well known that (Helliwell, 1965; Kennel \& Petschek, 1966; Inan, 1977; Chang, 1983) the only electrons that will fall into the lower ionosphere i.e. into the loss cone, are the ones which satisfy the inequality, where $\alpha$ is any pitch angle of electron and, the half loss cone pitch angle which is measured by the following expression

$$
\sin ^{2} \alpha_{0}=\frac{E_{m}^{3}}{L^{2}\left(4 L^{2}-3 E_{m} L\right)^{1 / 2}}
$$

Where,

$$
E_{\text {mit }}=\frac{R_{0}-H_{\text {m }}}{R_{0}}
$$


Where $\mathrm{Hm}$ is the mirror height, the height from where near the earth surface the gyrating electron mirrors back to the equator from North Pole side to the equator to South Pole side. So we can say that the electron travels between mirror height in the first hemisphere, equatorial region, and mirror height in the second hemisphere inside the radiation belt that is why we call them trapped particles.

For proper discussion of wave-particle interaction i.e. pitch angle scattering of an energetic electron by ELF/VLF waves, we must know the loss cone pitch angle at considered L shells. Since we are studying wave-particle interaction in the inner radiation belt at $\mathrm{L}=1.2-1.8$, we compute loss cone pitch angle at these L shells (Table 2).

Table 2. Variation of loss cone pitch angle $\alpha \mathrm{o}$ with various $L$ shell values.

\begin{tabular}{|ll|}
\hline $\begin{array}{l}\text { McIlwain } \\
\text { Parameter }\end{array}$ & $\begin{array}{l}\text { Loss cone pitch } \\
\text { angle } \\
\text { L }\end{array}$ \\
\hline 1.1 & $\alpha_{0}$ (radians) \\
1.3 & 1.007 \\
1.5 & 0.658 \\
1.7 & 0.488 \\
\hline
\end{tabular}

These values are taken to be at a mirror height of $100 \mathrm{~km}$ i.e. the base of the ionosphere. Consideration of $\mathrm{Hm}$ to be 110 $120 \mathrm{~km}$ changes values slightly (of the order of $3 \times 10-4)$ bringing almost no change in $\alpha o$ values.

\subsection{Resonant velocity and resonant energy}

The resonant velocity of the electrons for different frequencies at $\mathrm{L}=1.2$ is given in Table 3.

Table 3. Variation of $\mathrm{V} 11 / \mathrm{c}$ and $\mathrm{Er}$ at interacting frequencies at $\mathrm{L}=1.2$

\begin{tabular}{|lll|}
\hline $\mathrm{f}(\mathrm{kHz})$ & $\mathrm{V}_{\mathrm{I}} / \mathrm{c}$ & $\mathrm{E}_{\mathrm{I}}(\mathrm{MeV})$ \\
\hline 1 & 0.9980 & 7.624 \\
2 & 0.9956 & 4.936 \\
3 & 0.9931 & 3.857 \\
4 & 0.9907 & 3.238 \\
5 & 0.9882 & 2.830 \\
\hline
\end{tabular}

The frequency range $\mathrm{f}=1-3 \mathrm{kHz}$ represents ELF waves whereas $\mathrm{f}=3-5 \mathrm{kHz}$ represents VLF waves. We see that as frequency increases resonating velocity of the electrons decreases, implying an inversely proportional relationship between the aforementioned parameters. The same happens for resonant energy also and hence it is maximum at 1 $\mathrm{kHz}$ frequency and minimum at $5 \mathrm{kHz}$ frequency. The corresponding values (for $\mathrm{L}$ $=1.4$ ) are given in Table 4 .

Table 4. Variation of $\mathrm{Vll} / \mathrm{c}$ and $\mathrm{Er}$ at interacting frequencies at $\mathrm{L}=1.4$.

\begin{tabular}{|lll|}
\hline $\mathrm{f}(\mathrm{kHz})$ & $\mathrm{V}_{\mathrm{Il}} / \mathrm{c}$ & $\mathrm{E}_{\mathrm{I}}(\mathrm{MeV})$ \\
\hline 1 & 0.9964 & 5.512 \\
2 & 0.9923 & 3.606 \\
3 & 0.9882 & 2.819 \\
4 & 0.9841 & 2.364 \\
5 & 0.9801 & 2.060 \\
\hline
\end{tabular}

Table 4, gives the resonant energy of the electrons taking part in the wave-particle interaction with whistler-mode waves in the magnetosphere of the inner radiation belt. It shows that $\mathrm{V}_{\mathrm{ll}} / \mathrm{c}$ and $\mathrm{E}_{\mathrm{r}}$ are directly proportional to $1 / \mathrm{f}$. We see that there comes a drop at $\mathrm{L}=1.1$ in respect of values at $\mathrm{L}$ shells from $L=1.2-1.7$ (Table 1). This is because electron density at $\mathrm{L}=1.1$ is $20,000 \mathrm{el} / \mathrm{cm}^{3}$ whereas at $\mathrm{L}=1.2$ it is $8130 \mathrm{el} / \mathrm{cm}^{3}$. Our explanation is satisfactory because whereas electron density suddenly decreases from L $=1.1-1.2$, the decrement of electron density between $L=1.2-1.7$ is slowly varying.

Now we study the variation of resonant energy of electrons participating in waveparticle interaction in the inner radiation belt at different $\mathrm{L}$ shells for frequencies at 3 and 5 $\mathrm{kHz}$ Figure 1. It can be noticed that the value of resonance energy increases at $\mathrm{L}=1.2$. There is a decrease in the value of resonant energy at $\mathrm{L}=1.3$ and other $\mathrm{L}$ shells. This is also due to $20,000 \mathrm{el} / \mathrm{cm}^{3}$ plasma density at $\mathrm{L}=1.1$ as discussed earlier.

3.3 Pitch angle diffusion coefficient and Lifetime of electrons

Now we compute the pitch-angle diffusion 
coefficient $\mathrm{D}(\alpha)$ at $\mathrm{L}=1.2$ for the frequencies considered above. We see that as the frequency increases diffusion coefficient decreases. It means that ELF waves are more efficient than VLF waves to dump the highenergy electrons in the radiation belt into the ionosphere. In this case, we have taken wave spectral density to be $1 \mathrm{pT}^{2} / \mathrm{Hz}$. If we consider wave's spectral density lesser or greater by a factor of 10 , the diffusion coefficient $\mathrm{D}(\alpha)$ follows in the same manner i.e. for $0.1 \mathrm{pT}^{2} /$ $\mathrm{Hz}$ we have obtained a diffusion coefficient $\mathrm{D}(\alpha)$ value smaller by a factor of 10 as compared to the one computed for $1 \mathrm{pT}^{2} / \mathrm{Hz}$. But for $10 \mathrm{pT}^{2} / \mathrm{Hz}$ we get a higher diffusion coefficient $D(\alpha)$ value which is 10 times the values computed at $1 \mathrm{pT}^{2} / \mathrm{Hz}$. Now for $1 \mathrm{pT}^{2} / \mathrm{Hz}$ only, we see the variation of pitch angle diffusion coefficient $\mathrm{D}(\alpha)$ at different L shells for ELF waves at frequency 1 $\mathrm{kHz}$ and VLF waves at frequency $5 \mathrm{kHz}$. We see that as the $\mathrm{L}$ shell value increases, the diffusion coefficient $D(\alpha)$ value also shows an increasing tendency Figure 2. Our results are supported by the ones obtained in previous studies (Singh 1991; Singh 1992; Sazhin 1991; Simoes et al., 2012).

Our consideration of wave spectral density range to be $0.1-10 \mathrm{pT}^{2} / \mathrm{Hz}$ is based on previous studies. Summers et al. (2008) have studied electron scattering by whistlermode ELF hiss in the plasmasphere. In this case, the electrons have been in the energy range of $0.1-1 \mathrm{Mev}$, with wave amplitude of $300 \mathrm{pT}$ to be maximum and less than $1 \mathrm{pT}$ wave amplitude to be minimum. Since we can easily find out wave spectral density to be $0.1-10 \mathrm{pT}^{2} / \mathrm{Hz}$.

We get a lifetime of the electrons as the inverse of diffusion coefficient $\mathrm{D}(\alpha)$ and find values at $\mathrm{L}=1.2,1.4$, and 1.6 for frequencies at $1-5 \mathrm{kHz}$, as 37.54 hours (a minimum for 1 $\mathrm{kHz}$ ) and 2.03 hours for $5 \mathrm{kHz}$ as minimum lifetime. The maximum lifetime for $1 \mathrm{kHz}$ and $5 \mathrm{kHz}$ are 227.68 hours and 13.95 hours respectively. Thus we can say that we are finding the lifetime values of electrons in the range of 2.03 hours, a minimum and 227.68 hours, a maximum for all frequencies Figure 3.

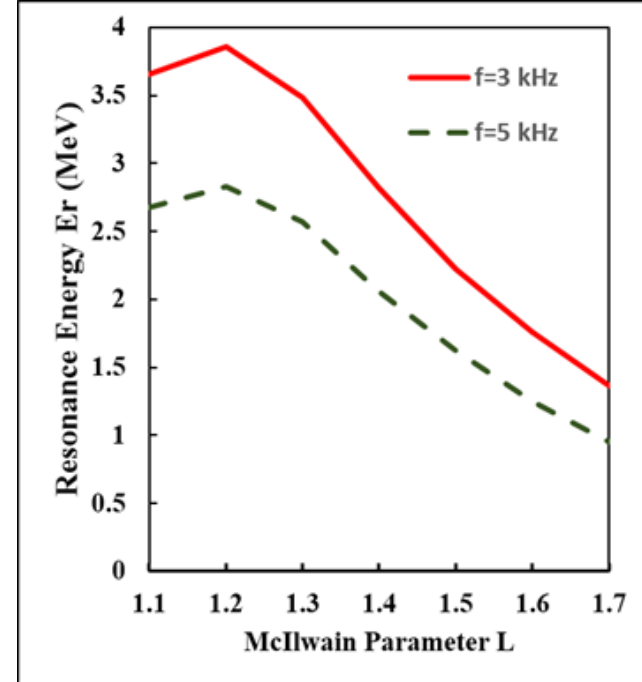

Fig. 1. Variation of Er values with different $\mathrm{L}$ shell values at constant frequency $\mathrm{f}=3$ and $5 \mathrm{kHz}$

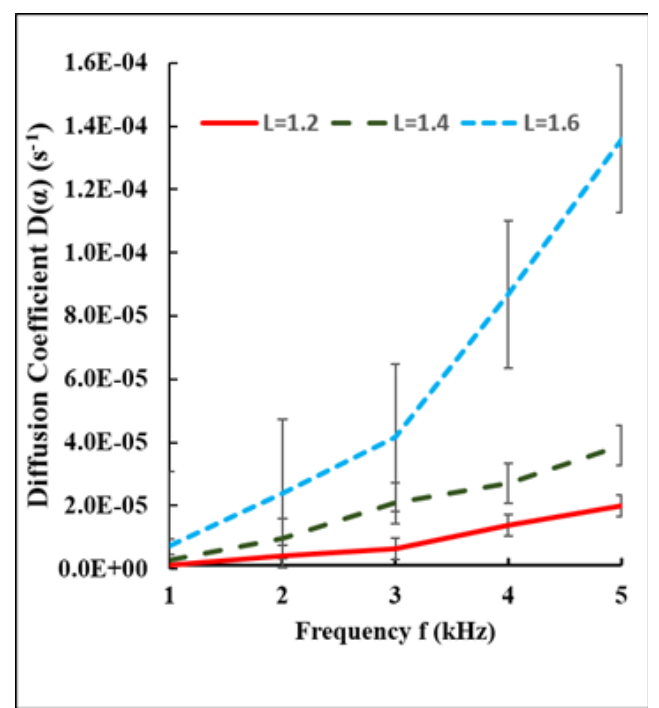

Fig. 2. Variation of Diffusion Coefficient $\mathrm{D}(\alpha)$ with frequency at different $\mathrm{L}$ shell values

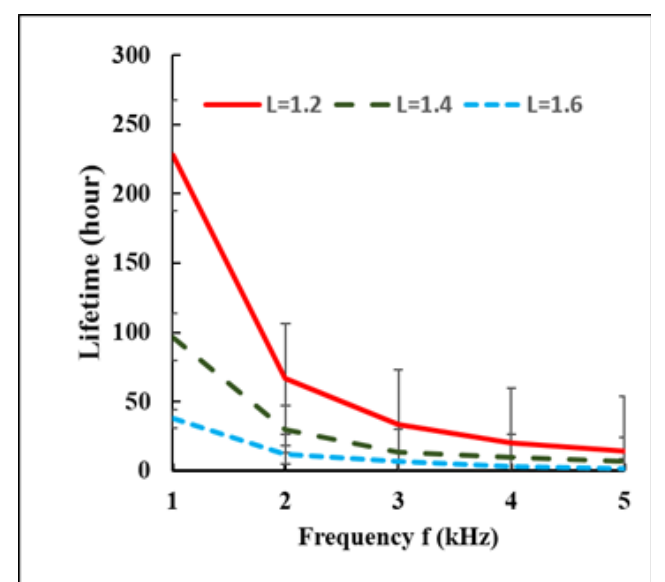

Fig. 3. Variation of Life Time with frequency at different $\mathrm{L}$ shells 
Our study is supported by the data collected aboard the Combined Release and Radiation Effect Satellite (CRRES) and analyzed by Summers et al. (2007a, b, 2008) and Shprits et al. (2007). Shprits et al. (2007) have reported the lifetimes of energetic electrons in the range of a day to hours at lower energies which is close to our calculation. Graf et al. (2009) have also shown the same results from Detection of Electro-Magnetic Emissions Transmitted from Earthquake Regions (DEMETER) transmitter analysis. The significance of the lifetime values of electrons obtained in this study are supported by Shao et al. (2009) who have shown that Earth's inner Van Allen radiation belt is filled with the stable flux of energetic particles and cyclotron resonance interaction is the process that can bring down the energetic particles into the lower ionosphere.

\section{ACKNOWLEDGMENTS}

The author is grateful to the Director and Management of the institute for providing facilities to research the department.

\section{References}

Angerami, J.J. \& Thomas, J.O. (1963). The distribution of ions and electrons in the earth's exosphere. Technical Report No. 3412_3, Stanford University Stanford.

Bortnik, J., Chen, L., li, W., Thorne, R.M. \& Horne, R.B. (2011). Modeling the evolution of chorus waves into a plasmaspheric hiss. Journal of Geophysical Research 116: A08221, doi:10.1029/2011/ A016499.

Chang, H.C., \& Inan, U.S. (1983). A theoretical model study of observed correlations between whistler-mode waves and energetic electron precipitation events in the magnetosphere. Journal of Geophysical Research 88: 10053-10064.
Chen, Y., Reeve, G. \& Reiner, F. (2007). The energization of relativistic electrons in the outer Van Allen radiation belt. Nature Physics 3: 614-617.

Daglis, I.A., Katsavrias, C. \& Georgiou, M. (2019). From solar sneezing to killer electrons: outer radiation belt response to solar eruptions. Philosophical Transactions of the Royal Society A, doi.org/10.1098/ rsta.2018.0097.

Graf, K.L., Inan, U.S., Piddyachig, D., Kulkarni, P., Parrot, M. \& Sauvaud, J.A. (2009). DEMETER observations of transmitter-induced precipitation of inner radiation belt electrons. Journal of Geophysical Research 114: doi:10.1029/2008JA013949.

Helliwell, R.A. (1965). Whistler and related ionospheric phenomena. Stanford University Press.

Inan, U.S. (1977). Nonlinear gyroresonant interactions of energetic particles and coherent VLF waves in the magnetosphere. Technical Report No. 3414-3, Starlab, Stanford University.

Inan, U.S., Bell, T.F. \& Helliwell, R.A. (1978). Nonlinear pitch angle scattering of energetic electrons by coherent VLF waves in the magnetosphere. Journal of Geophysical Research 83: 3235.

Kennel, C.F. \& Petschek, H.E. (1966). Limit on stably trapped particle fluxes. Journal of Geophysical Research 71:1.

Potapov, A., Ryzhakova L. \& Tsegmed, B. (2016). A new approach to predict and estimate enhancements of killer electron flux at geosynchronous orbit. Acta Astonautica 126: $47-51$. 
Prasad, S.S. (1968). Nighttime ionic composition and temperature over Arecibo. Journal of Geophysical Research 73: 6795.

Sazhin, S.S. (1991). Pitch angle diffusion at low L shells is always weak. Indian Journal of Radio and Space Physics 20: 446.

Sentman, D.D. \& Goertz, C.K. (1978). Whistler mode noise in Jupiter's inner magnetosphere. Journal of Geophysical Research 83: 3151.

Shao, X., Papadopoulos, K. \& Sharma, A.S. (2009). Control of energetic proton flux in the inner radiation belt by artificial means. Journal of Geophysical Research 114: A07214, doi:10.1029/2009 JAD 14066.

Shprits Y., Kondrashov, D., Chen, Y., Thorne, R., Ghil, M. et al., (2007). Reanalysis of relativistic radiation belt electron fluxes using CRRES satellite data, a radial diffusion model, and a Kalman filter. Journal of Geophysical Research 112: doi:10.1029/2007JA012579.

Simoes, F., Pfaff, R., Berthelier, J.J. \& Klenzing, J. (2012). A review of lowfrequency electromagnetic wave phenomena related to tropospheric - ionospheric coupling mechanisms. Space Science Reviews 168: 551-93.

Singh, D.P. (1991). Strong diffusion of resonant electrons by VLF waves. Indian Journal of Radio and Space Physics 20: 424.

Singh, D.P. (1992). L dependence of trapped electron diffusion by ELF waves. Indian Journal of Radio and Space Physics 21: 250.

Singh, D.P. \& Singh, U.P. (2004). The diffusion coefficient and wave amplification determination from pitch angle-dependent kinetic energy of electrons. Indian Journal of Radio and Space Physics 33: 234-40.
Singh, D.P. \& Singh, U.P. (2006). An explanation of non-observation of transmitted VLF signals on the ground. Journal of Atmospheric and Solar-Terrestrial Physics 68: 832-837.

Singh, U.P. (1993). Whistlers / VLF emissions and related phenomena. Ph.D. thesis, Banaras Hindu University, Varanasi, India.

Singh, U.P. \& Singh, D.P. (1998). Intense low latitude VLF emissions observed aboard Ariel 4. Journal of Geophysical Research 103: 20727-20733.

Summers, D. Ni, B. \& Meredith, M.T. (2007a). Time scales for radiation belt electrons acceleration and loss due to resonant wave-particle interaction. Journal of Geophysical Research 112: Doi;10.1029/2006 J A 011801.

Summers, D., Ni, B. \& Meredith, M.T. (2007b). Evaluation for VLF chorus, ELF hiss, and electromagnetic ion cyclotron. Journal of Geophysical Research 112: Doi;10.1029/2006 J A 011993.

Summers, D., Ni, B. \& Meredith, M.T. (2008). Electron scattering by whistler-mode ELF hiss in plasmaspheric plumes. Journal of Geophysical Research 113: Doi;10.1029 /2007 JA 012678.

Vranjes, J. \& Tanaka, M.Y. (2005). On the gravity-induced electric field in space plasmas. Physica Scripta 71: 325-328.

Submitted: $\quad 26 / 04 / 2020$

Revised: $\quad 23 / 11 / 2020$

Accepted: $\quad 24 / 11 / 2020$

DOI: $\quad 10.48129 /$ kjs.v48i4.9620 